University of Wollongong

Research Online

Faculty of Engineering and Information

Faculty of Engineering and Information

Sciences - Papers: Part A

Sciences

$1-1-2013$

Measurement of multi-slice computed tomography dose profile with the Dose Magnifying Glass and the MOSkin radiation dosimeter

C P. L Lian

University of Wollongong, pll098@uowmail.edu.au

J H. D Wong

University of Wollongong, jwong@uow.edu.au

A Young

St Vincent's Hospital Sydney

D Cutajar

University of Wollongong, deanc@uow.edu.au

M Petasecca

University of Wollongong, marcop@uow.edu.au

See next page for additional authors

Follow this and additional works at: https://ro.uow.edu.au/eispapers

Part of the Engineering Commons, and the Science and Technology Studies Commons

Research Online is the open access institutional repository for the University of Wollongong. For further information contact the UOW Library: research-pubs@uow.edu.au 


\title{
Measurement of multi-slice computed tomography dose profile with the Dose Magnifying Glass and the MOSkin radiation dosimeter
}

\begin{abstract}
This study describes the application of two in-house developed dosimeters, the Dose Magnifying Glass (DMG) and the MOSkin dosimeter at the Centre for Medical Radiation Physics, University of Wollongong, Australia, for the measurement of CT dose profiles for a clinical diagnostic 16-slice MSCT scanner. Two scanner modes were used; axial mode and helical mode, and the effect of varying beam collimation and pitch was studied. With an increase in beam collimation in axial mode and an increase of CT pitch in helical mode, cumulative point dose at scanner isocentre decreased while FWHM increased. There was generally good agreement to within $3 \%$ between the acquired dose profiles obtained by the DMG and the film except at dose profile tails, where film over-responded by up to $30 \%$ due to its intrinsic depth dose dependence at low doses.
\end{abstract}

\section{Keywords}

moskin, glass, magnifying, profile, dose, tomography, dosimeter, computed, radiation, slice, multi, measurement

\section{Disciplines}

Engineering | Science and Technology Studies

\section{Publication Details}

Lian, C. P. L., Wong, J. H. D., Young, A., Cutajar, D., Petasecca, M., Lerch, M. L. F. \& Rosenfeld, A. B. (2013). Measurement of multi-slice computed tomography dose profile with the Dose Magnifying Glass and the MOSkin radiation dosimeter. Radiation Measurements, 55 51-55.

\section{Authors}

C P. L Lian, J H. D Wong, A Young, D Cutajar, M Petasecca, M L. F Lerch, and Anatoly B. Rosenfeld 


\title{
Measurement of Multi-Slice Computed Tomography Dose Profile with the Dose Magnifying Glass and the MOSkin Radiation Dosimeter

\author{
C.Lian ${ }^{\text {a,* }}$, J. Wong ${ }^{\text {a,b }}$, A. Young ${ }^{\text {c }}$, D. Cutajar ${ }^{\mathrm{a}}$, M. Petasecca ${ }^{\mathrm{a}}$, M. Lerch $^{\mathrm{a}}$, A. Rosenfeld $^{\mathrm{a}}$
} \\ ${ }^{a}$ Centre for Medical Radiation Physics, University of Wollongong, Wollongong NSW 2522, Australia \\ *Corresponding author Tel: + 6124221 3507; Fax: +612 42215474 Email address: pll098@uowmail.edu.au \\ ${ }^{\mathrm{b}}$ Department of Biomedical Imaging, Faculty of Medicine Building, University of Malaya, 50603 Kuala Lumpur, Malaysia \\ 'St Vincent's Hospital, Department of Nuclear Medicine and PET, Sydney NSW 2010, Australia
}

\begin{abstract}
This study describes the application of two in-house developed dosimeters, the Dose Magnifying Glass (DMG) and the MOSkin dosimeter at the Centre for Medical Radiation Physics, University of Wollongong, Australia, for the measurement of CT dose profiles for a clinical diagnostic 16-slice MSCT scanner. Two scanner modes were used; axial mode and helical mode, and the effect of varying beam collimation and pitch was studied. With an increase in beam collimation in axial mode and an increase of CT pitch in helical mode, cumulative point dose at scanner isocentre decreased while FWHM increased. There was generally good agreement to within $3 \%$ between the acquired dose profiles obtained by the DMG and the film except at dose profile tails, where film over-responded by up to $30 \%$ due to its intrinsic depth dose dependence at low doses.
\end{abstract}

Keywords: CT dosimetry; MOSFET; DMG. 


\section{Measurement of Multi-Slice Computed Tomography Dose Profile with the Dose Magnifying Glass and the MOSkin Radiation Dosimeter}

\section{Introduction}

$\mathrm{CTDI}_{100}$, an existing dose quality assurance measure of CT scanner output, is defined as the average dose to a cylindrical homogeneous PMMA phantom, from a single axial scan with integration limits of $\pm 50 \mathrm{~mm}$, described by Equation 1 .

$$
\left.C T D I_{100}=\frac{1}{T} \int_{z_{2}}^{z_{1}} D(z) d z \quad(\mathrm{mGy}) \quad \text { (Eq. } 1\right)
$$

where $\mathrm{z}_{1=}-50$ and $\mathrm{z}_{2}=+50$ are the limits of integration in $\mathrm{mm}, \mathrm{D}(\mathrm{z})$ is the single slice dose profile and $\mathrm{T}$ is the nominal slice thickness in $\mathrm{mm}$.

$\mathrm{CTDI}_{100}$ has been known to severely underestimate modern, wide cone beam doses (International Atomic Energy Agency, 2011) due to considerable contribution of doses from dose tails extending beyond the conventional $100 \mathrm{~mm}$ CT pencil ion chamber length frequently used in clinical CT quality assurance (DCT10, RTI Electronics). Recently, several authors have also reported on the enlarged longitudinal beam width (up to $160 \mathrm{~mm}$ ) associated with the latest cone beam CT scanners (Geleijns et al., 2009; Gomà et al., 2011; Lin and Herrnsdorf, 2010; Mori et al., 2005; Nakonechny et al., 2005). It follows that besides the use of longer phantoms, a new kind of dosimetry tool and methodology is necessary for more accurate assessment of doses associated with these new wide cone beam CT scanners.

The present work is a proof of concept study towards improved wide beam MSCT dose assessment using the Dose Magnifying Glass (DMG) calibrated against the MOSkin dosimeter. The first aim of the study was to map the longitudinal z-axis CT dose profile in the CT scanner axial mode with different beam collimations $(1.25 \mathrm{~mm}, 5 \mathrm{~mm}, 20 \mathrm{~mm})$ and in the helical mode with different CT pitch values $(0.625,0.875,1.35)$. The second aim of the study was to demonstrate the over-ranging phenomenon associated with CT helical scans compared to CT axial scans. 


\section{Methodology}

\subsection{CT scanner and dosimeters}

The CT system used was a 16-slice General Electric (GE) Discovery 670 NM/CT SPECT/CT scanner. Table 1 shows the scan parameters used for this study which were designed based on commonly used clinical imaging parameters for diagnostic CT scans.

Table 1: Scan parameters of this study with a typical clinical CT tube potential of $120 \mathrm{kVp}$ tube current of $300 \mathrm{mAs}$ and a total scan range of $120 \mathrm{~mm}$.

\begin{tabular}{llccl}
\hline $\begin{array}{l}\text { CT } \\
\begin{array}{l}\text { Scan } \\
\text { mode }\end{array}\end{array}$ & $\begin{array}{l}\text { CT Detector } \\
\text { configuration }\end{array}$ & $\begin{array}{l}\text { Nominal beam } \\
\text { collimation } \\
(\mathbf{m m})\end{array}$ & Scan length $(\mathbf{m m})$ & Pitch \\
\hline Axial & $2 \times 0.625 \mathrm{~mm}$ & 1.25 & 29.375 & - \\
& $4 \times 1.25 \mathrm{~mm}$ & 5 & 30 & - \\
& $8 \times 2.5 \mathrm{~mm}$ & 20 & 30 & - \\
\hline & & & 30 & 0.625 \\
Helical & $8 \times 2.5 \mathrm{~mm}$ & 20 & & 0.875 \\
& & & 30.35 \\
\hline
\end{tabular}

The MOSkin dosimeter, designed and developed at the Centre for Medical Radiation Physics (CMRP), University of Wollongong, Australia, has previously been investigated for applications in radiation therapy (Hardcastle et al., 2008; Kwan et al., 2009) and recently in CT (Lian et al., 2012). It was found to be tissue equivalent at depth in clinical kilovoltage beams (Lian et al., 2011) and was therefore selected as our gold standard absolute dosimeter in this work.

The DMG is a high-spatial resolution relative dosimeter developed at the CMRP. The DMG comprises an array of $128 \mathrm{n}^{+}$strips fabricated on a p-type silicon substrate of $375 \mu \mathrm{m}$ thickness. Each $\mathrm{n}^{+}$strip is a silicon diode with width $20 \mu \mathrm{m}$ and length $2 \mathrm{~mm}$, wire-bonded onto kapton. An electronic system allows simultaneous real time readout of its 128 channels. Figures 1 and 2 illustrate the topological schematic diagram and photographs of the DMG respectively. Further details on its construction, design and applications in clinical therapy can be found in (Hardcastle et al.,2012 and Wong et al.(2010, 2011) )

In this study, the beam profile data acquired by the DMG was compared with the dose profile obtained with a new type of Gafchromic film, XR-QA2 film (International Specialty Products, 2011), specially developed for dosimetry in radiology. 


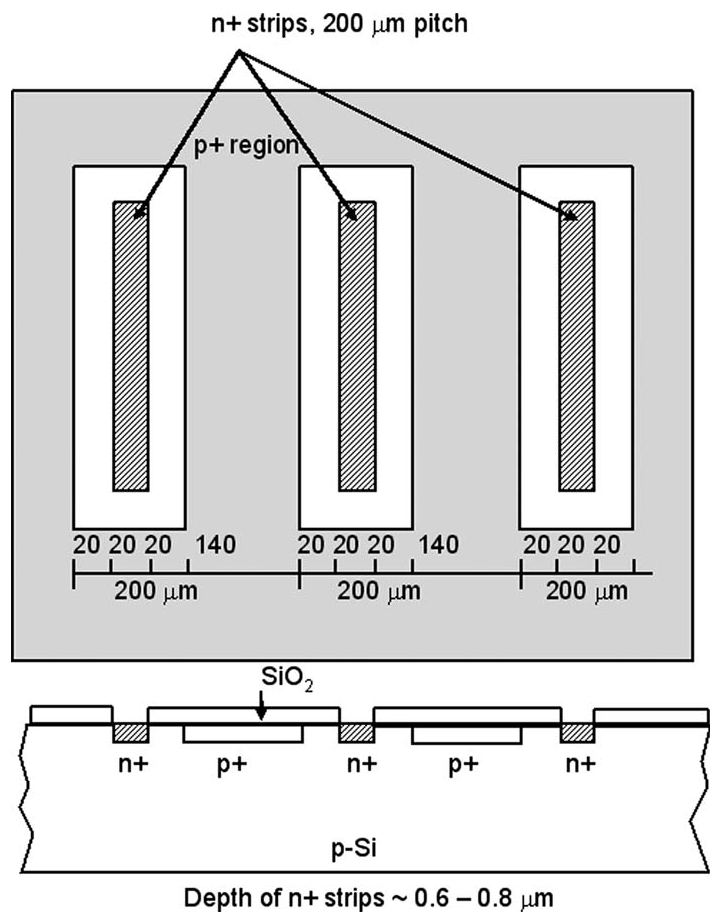

Figure 1: Topology of DMG, not-to-scale (From Wong et al 2010).

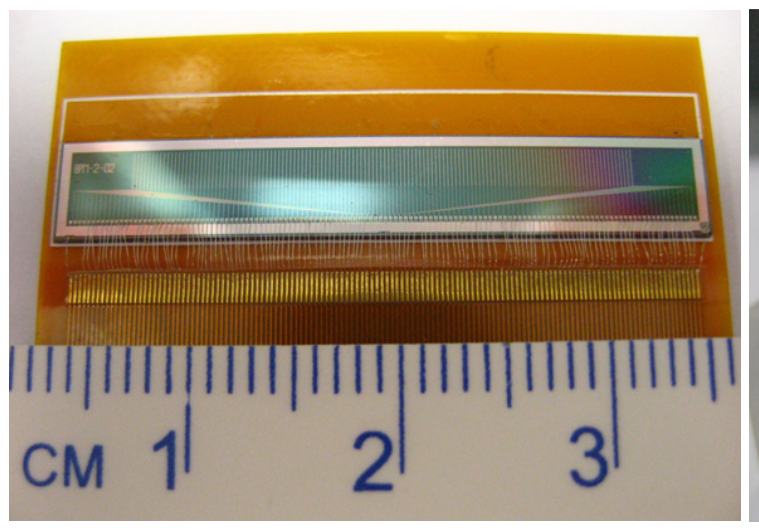

(a)

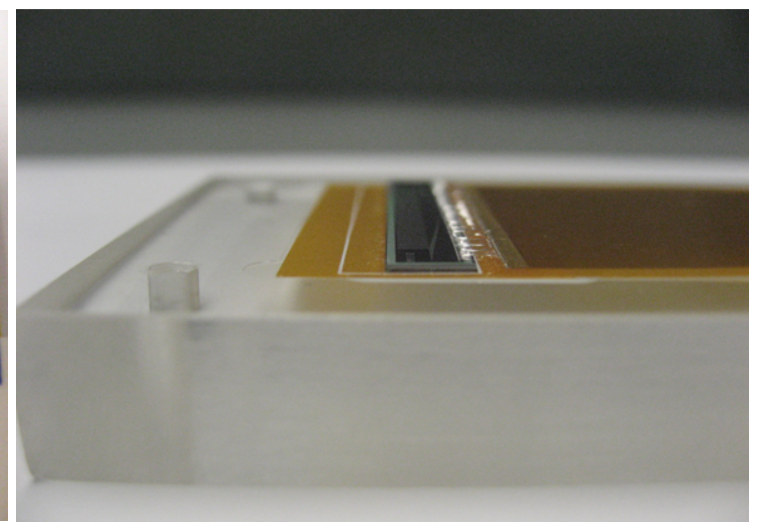

(b)

Figure 2: Photograph of (a) the $25.6 \mathrm{~mm}$ silicon detector array of the Dose Magnifying Glass (DMG) (b) DMG mounted on $1 \mathrm{~cm}$ thick solid water with its connector at one end which can be inserted into a phantom.

\subsection{Experimental setup}

Figure 3 illustrates our experimental setup. The DMG was placed between seven slabs of 1 cm thick solid water blocks (RMI 457 Gammex, Middleton, WI, USA), each of $30 \mathrm{~cm} \mathrm{x} 30$ $\mathrm{cm}$ dimensions. In addition, the DMG was surrounded by $1 \mathrm{~cm}$ thick blocks of solid water. The longitudinal axis of the silicon diode array was in coincidence with the z-axis of the CT 
scanner. The DMG was connected by two long ribbon cables to a computerized DAQ system outside of the CT room for real-time data acquisition and processing.

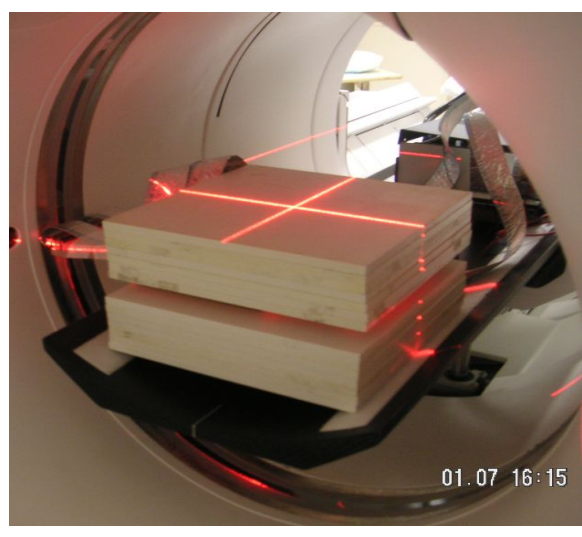

(a)

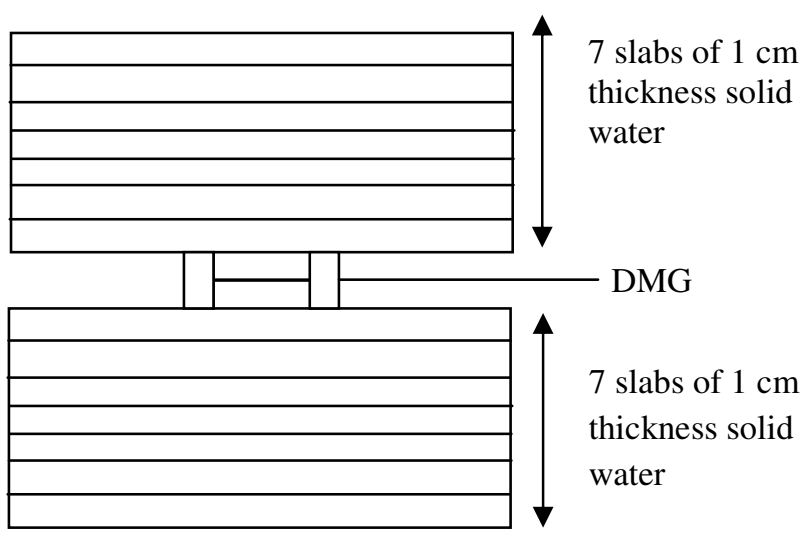

(b)

Figure 3: Experimental setup of the CMRP DMG system. (a) Photograph showing the DMG aligned to the isocentre of the CT scanner using the laser positioning reference markers. (b) Side view of DMG placed between solid water slabs, $8 \mathrm{~cm}$ above and below the sensitive detector array of the DMG.

\subsection{Dosimeter Calibration}

With the DMG set up as shown in Figure 3, the relative sensitivity response of each channel of the DMG was obtained with the $120 \mathrm{kVp} \mathrm{CT}$ beam (HVL=0.8 $\mathrm{mm} \mathrm{Cu}$ ) in scout mode (stationary x-ray tube), at a nominal beam width of $20 \mathrm{~mm}$ over a longitudinal total z-axis scan distance of $120 \mathrm{~mm}$. The DMG was manually shifted and re-positioned 6 times, in a sonamed "shift-and-measure" technique from $-60 \mathrm{~mm}$ to $+60 \mathrm{~mm}$ along the longitudinal z-axis of the CT scan couch, first described in Wong et al. (2011). The shift-and-measure technique was applied in the calibration to create a flat calibration x-ray CT field for all 128 channels of the detector as much as possible in this study.

After equalization of the 128 channels of the $25.6 \mathrm{~mm}$ length DMG diode array in the CT scout scan mode, the DMG sensitivity response was found to have a $3.6 \%$ coefficient of variation $(\mathrm{COV})$ in a single scan. With 3 repeated $\mathrm{x}$-ray $\mathrm{CT}$ scans, an average COV of $7 \%$ was obtained in the overall plotted $\mathrm{CT}$ dose profile. We attribute this overall variation to the reproducibility of the $\mathrm{CT}$ beam and the uncertainties associated with DMG positioning alignment.

The MOSkin dosimeter was separately calibrated in a superficial $150 \mathrm{kVp}$ x-ray beam $(\mathrm{HVL}=0.6 \mathrm{~mm} \mathrm{Cu})$ on a Gulmay D3300 superficial/orthovoltage therapy unit on the surface of a $30 \mathrm{~cm}$ x $30 \mathrm{~cm}$ x $20 \mathrm{~cm}$ solid water phantom. At this beam quality, the MOSkin calibration factor obtained was $0.67 \mathrm{mV} / \mathrm{mGy}$. 
Similarly, for the XR-QA2 film, small film pieces of $3 \mathrm{~cm} \times 3 \mathrm{~cm}$ each were cut to dimensions, and calibrated on the Gulmay D3300 therapy unit at $150 \mathrm{kVp}$ tube potential $(\mathrm{HVL}=0.6 \mathrm{~mm} \mathrm{Cu})$ on the surface of a $30 \mathrm{~cm} \times 30 \mathrm{~cm} \times 20 \mathrm{~cm}$ solid water phantom. Doses ranging from 0 to $200 \mathrm{mGy}$ were applied and the exposed films were digitized with a scanner (Microtek ScanMaker i800, 9600 x 4800 DPI CCD, 48-bit color) at least 24 hours after x-ray irradiation. Each film piece was scanned before and after the exposure at the same position on the scanner bed to ensure readout measurement consistency. In addition, the scanner was allowed to warm up at least 10 minutes prior to its first scan.

The images obtained from film digitization were analysed with ImageJ software (National Institutes of Health, MD) and Photoshop software. For each film piece, a central region of interest (ROI) of $1 \mathrm{~cm} \times 1 \mathrm{~cm}$ dimensions was specified and analysed. The following film scan protocol was applied: Reflective scan mode, RGB mode 48-bit color, resolution 300 dpi, $100 \%$ image scaling, with no color correction nor filters applied. Each film piece was scanned three times to minimize scanner response variations. The red channel was applied for image analysis. Finally, a calibration curve was plotted to determine the relationship between absorbed doses to optical density (i.e. degree of film darkening after irradiation).

\subsection{Depth dose characterization}

Figure 4 shows the comparison of the dose measured with MOSkin, Gafchromic XR-QA2 film and Marcus parallel plate ionization chamber (IC) at different depths in a 30 x $30 \mathrm{~cm}$ square solid water phantom measured in a $150 \mathrm{kVp}(0.6 \mathrm{~mm} \mathrm{Cu} \mathrm{HVL})$ superficial therapy beam on a Gulmay D3300 superficial therapy machine. The MOSkin dosimeter was reproducible to within $3 \%$ of ion chamber measurements for doses at depths larger than 80 $\mathrm{mm}$ in solid water. At depths larger than $80 \mathrm{~mm}$, the XR-QA2 film was found to overrespond by up to $30 \%$ compared to the IC and the MOSkin dosimeter. For depths less than $80 \mathrm{~mm}$, the average agreement between MOSkin and film was 4\%. For depths close to and at the surface with steep dose gradients (dose buildup), the larger disparity between MOSkin and film readouts was attributed to the different Water Equivalent Depth (WED) of the dosimeters. 


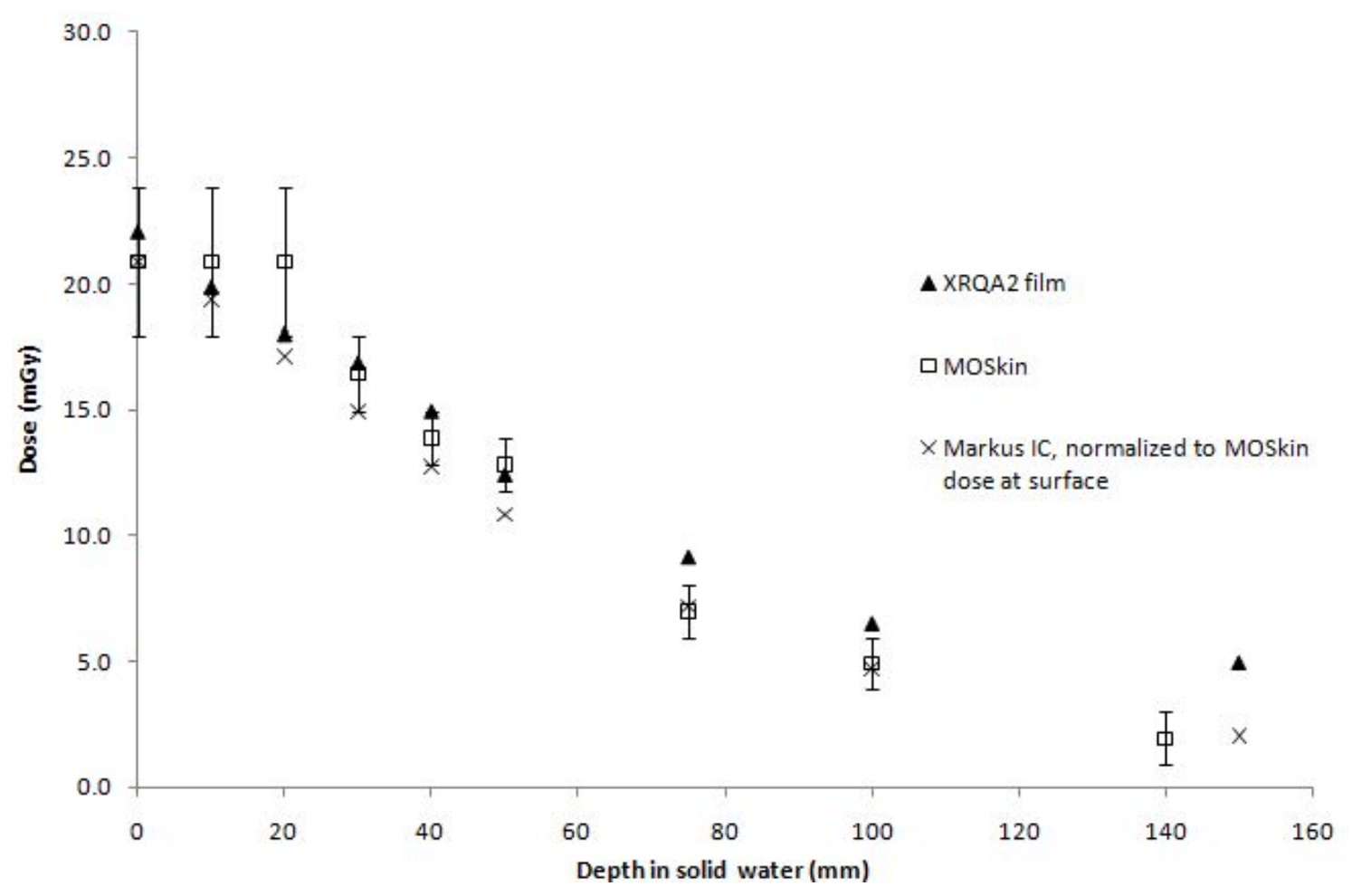

Figure 4: Low dose depth dose response of XR-QA2 film compared to the MOSkin dosimeter and MarkuS parallel plate ion chamber. $20 \mathrm{mGy}$ was delivered to the phantom surface at tube potential of $150 \mathrm{kVp}$ $(\mathrm{HVL}=0.6 \mathrm{~mm} \mathrm{Cu})$ with a Gulmay superficial/orthovoltage D3300 therapy unit.

\section{Results and Discussion}

\subsection{Axial scan mode}

For the axial scan mode (Figures 5 to 7$)$, the effect of increasing beam collimation $(1.25 \mathrm{~mm}$, $5 \mathrm{~mm}, 20 \mathrm{~mm}$ ) was studied. It was seen from the reconstructed dose profiles that with increasing beam collimation, FWHM increases while cumulative point dose measured at beam isocentre decreases. This may be explained by the spreading of the beam resulting in different scattering percentage contribution to cumulative central point dose.

MOSkin point dose and the film dose profiles generally agreed to within 3\%, and the dose profiles obtained with the film and DMG generally agreed except at the dose tails where the film over-responded by up to $30 \%$. From the depth dose characterization results presented in Figure 4, the observed $30 \%$ film over-response at the dose tails is to be expected. 


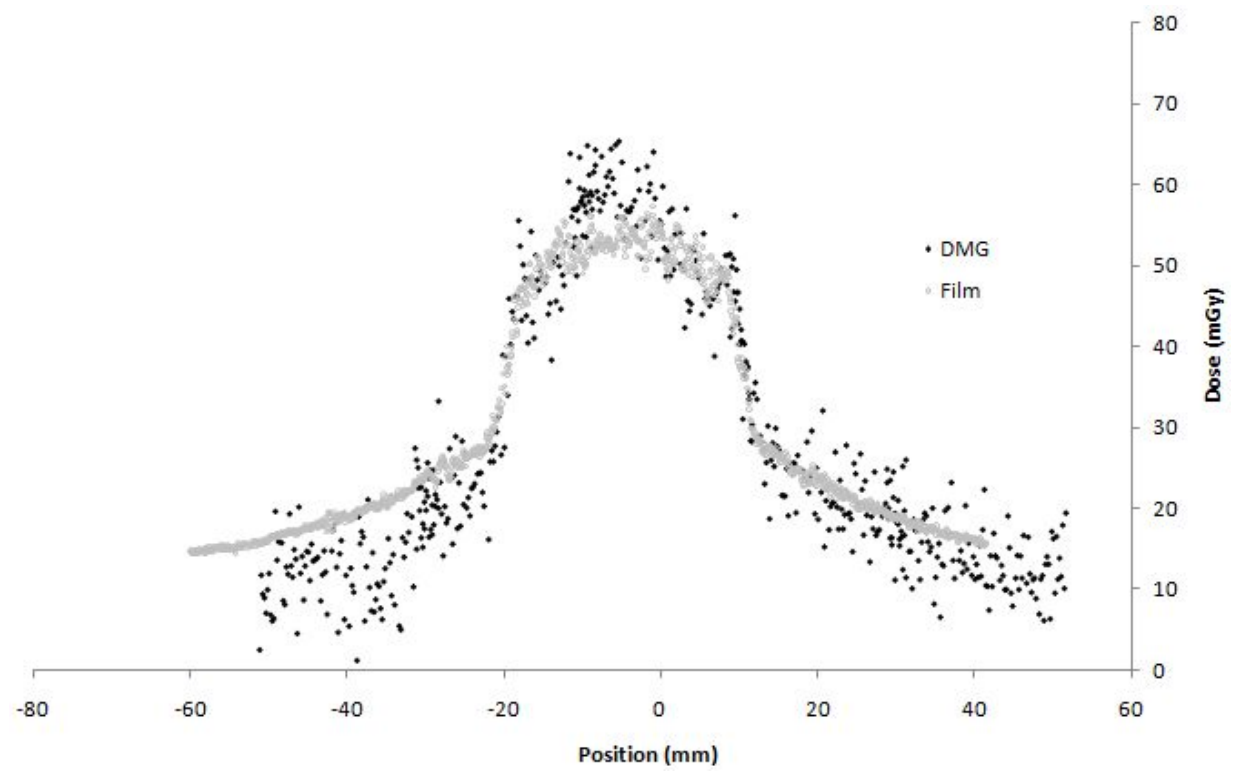

Figure 5: Axial scan mode with $1.25 \mathrm{~mm}$ nominal beam collimation. FWHM (DMG)=31.2 mm, FWHM $($ film $)=37.3 \mathrm{~mm}, \%$ difference in FWHM (film-DMG) $=16 \%$. MOSkin point dose measurement at beam isocentre $=55.1 \mathrm{mGy}$

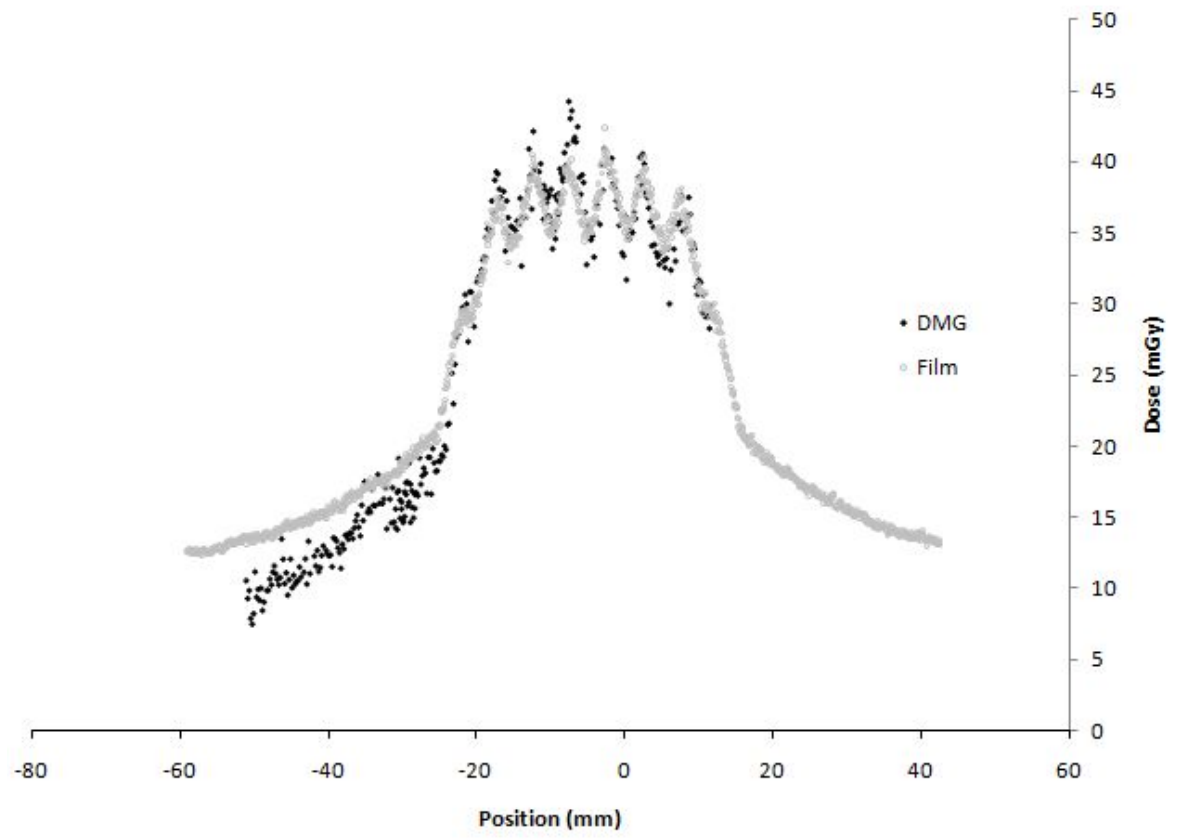

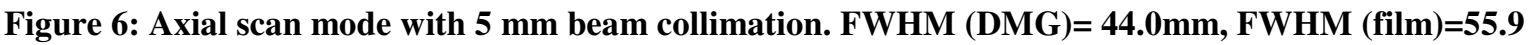
mm, \% difference in FWHM (film- DMG)= 21\%. MOSkin point dose measurement at beam isocentre= $36.2 \mathrm{mGy}$ 


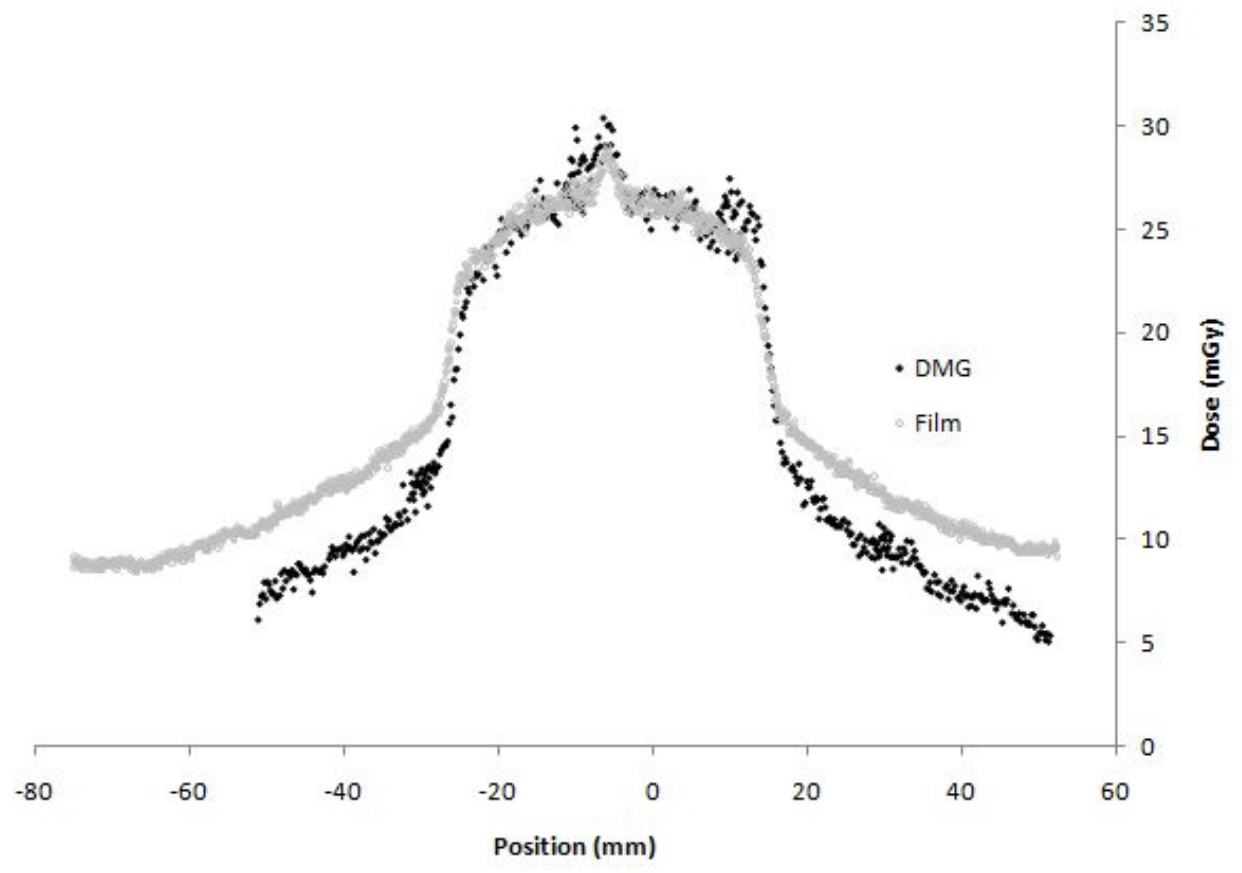

Figure 7: Axial scan mode with $20 \mathrm{~mm}$ beam collimation. FWHM (DMG)= 43.0 mm, FWHM (film) $=47.8$ $\mathrm{mm}$, \% difference in FWHM (film-DMG)=10.0\%. MOSkin point dose measurement at beam isocentre= 30.4 mGy

\subsection{Helical scan mode}

Figures 8 to 10 show the effect on dose profile with increasing CT pitch $0.625,0.875$ and 1.35. With an increase in $\mathrm{CT}$ pitch, the cumulative point dose measured by the MOSkin at beam isocentre decreased. This is to be expected since couch movement per rotation increases with collimation kept constant. Isocentre MOSkin point dose measurement agreed with film to within $3 \%$ for all helical scans.

The measured FWHM for the helical scan (Figure 8) was 1.5 times wider than that obtained for the axial scan (Figure 7) for the same $20 \mathrm{~mm}$ beam collimation. This result verifies the application of the DMG for the measurement of the known over-ranging effect in helical beams (Van der Molen and Geleijns, 2007). 


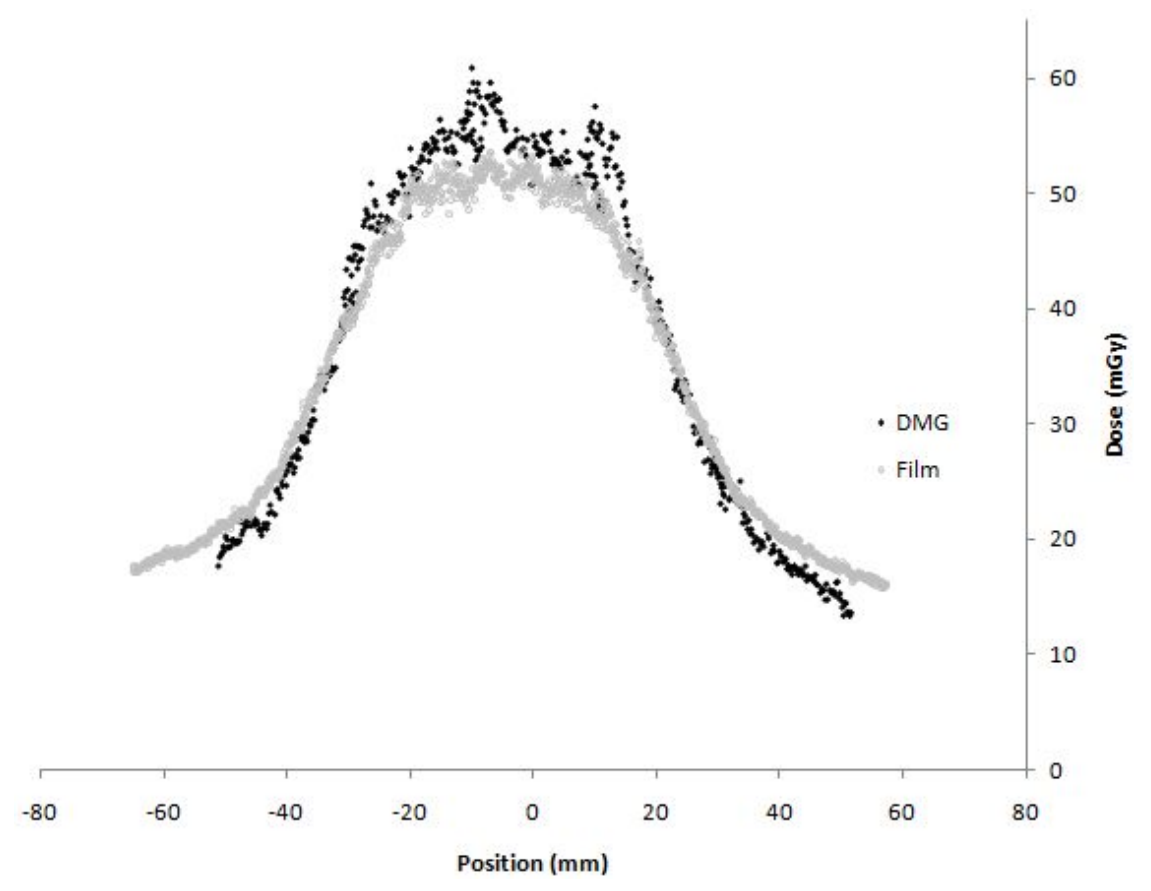

Figure 8: Helical scan mode at $20 \mathrm{~mm}$ nominal beam collimation, pitch 0.625. FWHM $($ DMG $)=61.4 \mathrm{~mm}$, FWHM (film) $=70.7 \mathrm{~mm}$, \% difference in FWHM (film-DMG)=13.0\%. MOSkin point dose measurement at beam isocentre $=55.1 \mathrm{mGy}$.

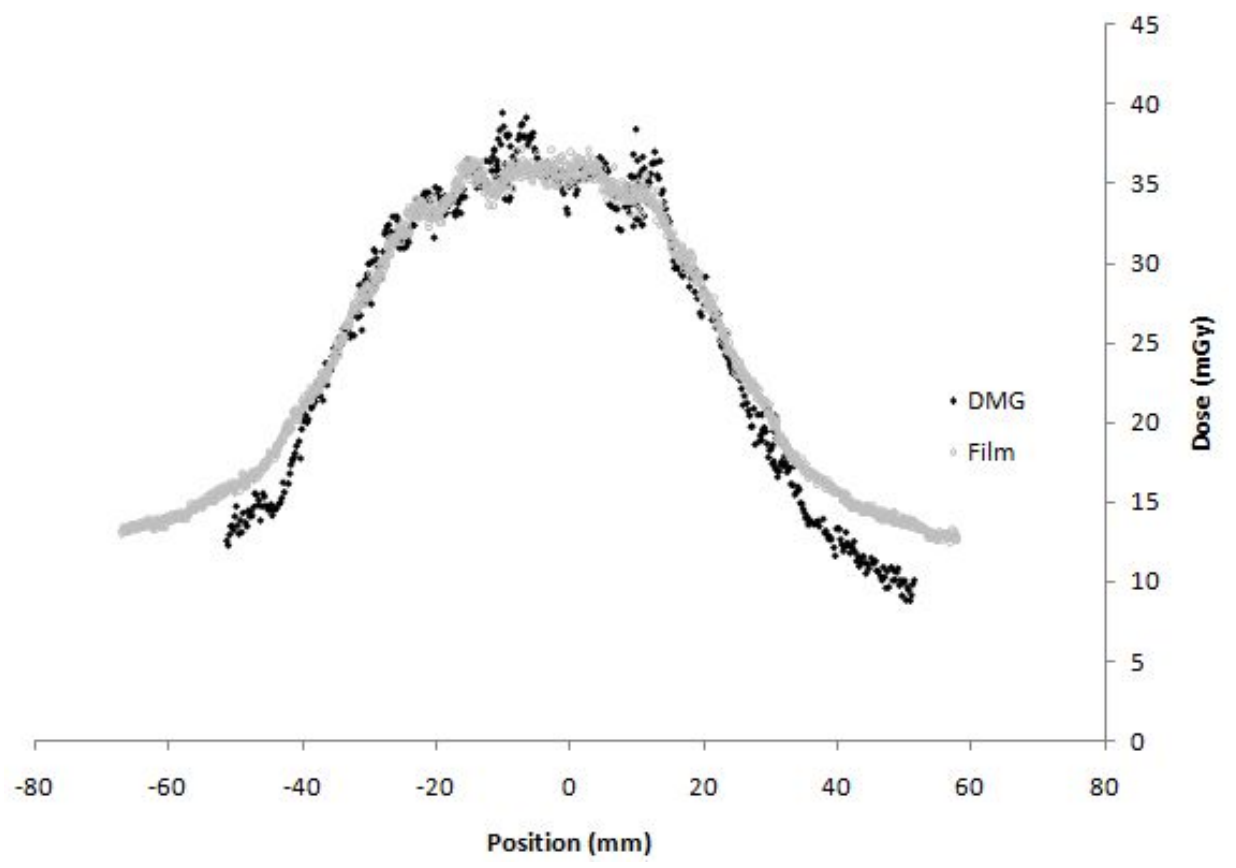

Figure 9: Helical scan mode at $20 \mathrm{~mm}$ nominal beam collimation, pitch 0.875 . FWHM $(\mathrm{DMG})=70.0 \mathrm{~mm}$, FWHM $($ film $)=74.6 \mathrm{~mm}$, \% difference in FWHM (film-DMG)=6.0\%. MOSkin point dose measurement at beam isocentre $=34.8 \mathrm{mGy}$ 


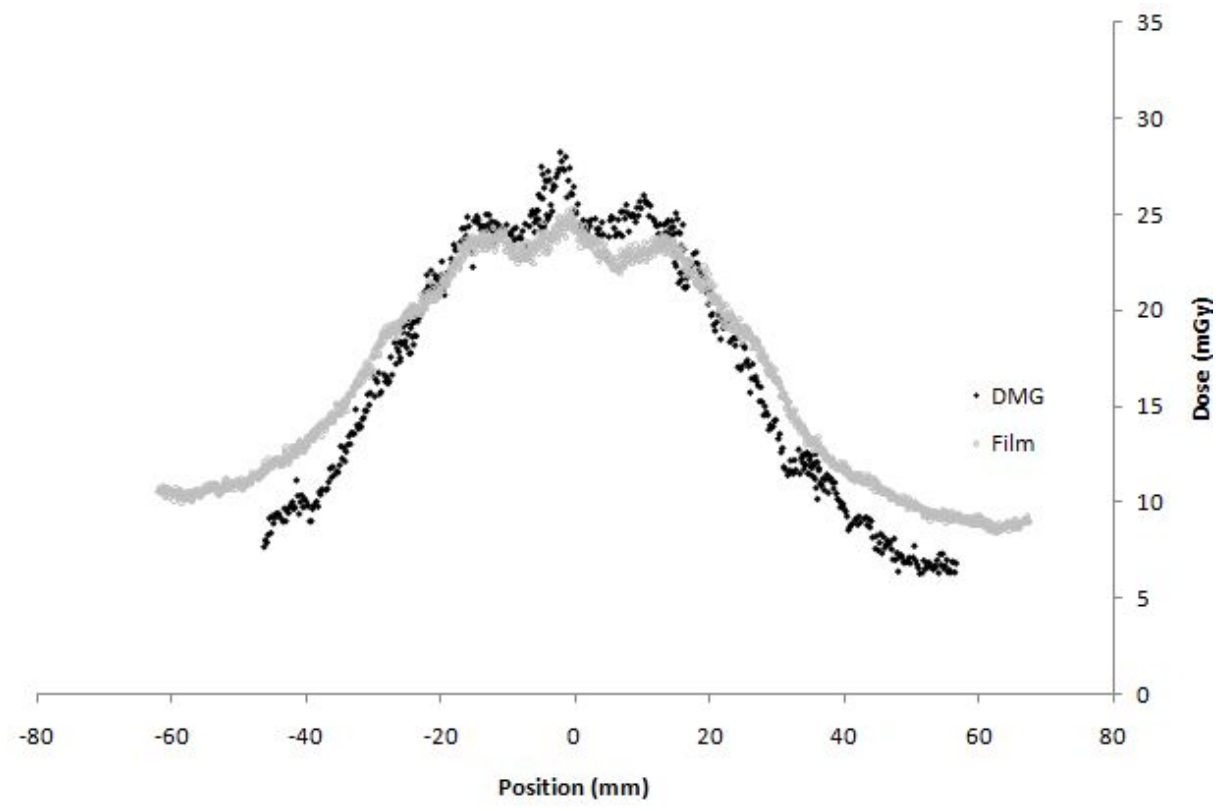

Figure 10: Helical scan mode at $20 \mathrm{~mm}$ nominal beam collimation, pitch 1.35. FWHM (DMG)= 61.3 mm, FWHM $($ film $)=78.5 \mathrm{~mm}$, \% difference in FWHM (film-DMG)=22.0\%. MOSkin point dose measurement at beam isocentre $=27.5 \mathrm{mGy}$

A major limitation in this study was the use of square solid water blocks surrounding the DMG instead of the conventional cylindrical $16 \mathrm{~cm}$ (head) or $32 \mathrm{~cm}$ (body)-diameter PMMA phantoms during CT dose scanning since square phantoms do not allow the correct assessment of the $\mathrm{CTDI}_{100}$. Moreover the current packaging of the DMG results in an asymmetrical response in comparison with that of a cylindrical phantom with the conventional CT pencil ion chamber placed in the central axis. However, the major objective of this study was a demonstration of a proof of concept, that the DMG calibrated by the MOSkin was feasible for MSCT dose profile acquisition. Verification of scanner CTDI 100 was beyond the scope of the present study.

It follows that future verification studies with the DMG should initially be focused on fabricating a custom cylindrical PMMA phantom that incorporates the DMG. This will then allow the direct comparison of $\mathrm{CTDI}_{100}$ measurements acquired with the DMG to that acquired by the conventional CT pencil-ion chamber for the further verification of narrow CT beam widths. Use of customised phantoms incorporating the DMG will also facilitate the future measurement of $\mathrm{CTDI}_{\infty}$ for wide cone beam widths. 


\section{Conclusion}

This study has shown the practical application of the DMG calibrated using the MOSkin dosimeter, for the measurement of diagnostic x-ray CT dose profiles in a typical 16-slice diagnostic x-ray MSCT scanner. The beam profile in the CT axial and helical scan modes were successfully acquired with the DMG. There was generally good agreement between the acquired dose profiles obtained by the DMG and the film to within 3\% except at the dose profile tails, where film over-responded by up to $30 \%$ due to the inherent depth dose response of film at low doses. The over-ranging phenomenon typically associated with helical scans compared to axial scans was also verified by the DMG and MOSkin measurements.

Based on the results obtained in this study, we recommend future application of both the MOSkin dosimeter and DMG for the dose profile assessment of longer beam widths associated with next generation wide cone beam CT scanners in order to overcome present limitations associated with the existing $\mathrm{CT}$ dose metric, $\mathrm{CTDI}_{100}$. Future work will be focused on fabricating a custom cylindrical phantom that incorporates the DMG.

\section{Acknowledgements}

The authors wish to thank Dr Martin Carolan for the use of the Gulmay D3300 superficial/ orthovoltage therapy unit for the calibration of the dosimeters used in this work.

\section{References}

Geleijns, J., Artells, M.S., Bruin, P.W.d., Mather, R., Muramatsu, Y., McNitt-Gray, M.F., 2009. Computed tomography dose assessment for a $160 \mathrm{~mm}$ wide, 320 detector row, cone beam CT scanner. Physics in Medicine and Biology 54.

Gomà, C., Ruiz, A., Jornet, N., Latorre, A., Pallerol, R., Carrasco, P., Eudaldo, T., Ribas, M., 2011. Radiation dose assessment in a 320-detector-row CT scanner used in cardiac imaging. Medical Physics 38, 1473-1480.

Hardcastle N, Bayliss A, Wong JH, Rosenfeld AB, WA., T., 2012. Improvements in dose calculation accuracy for small off-axis targets in high dose per fraction tomotherapy. Med Phys 39, 4788-4794.

Hardcastle, N., Soisson, E., Metcalfe, P., Rosenfeld, A., Tome, W., 2008. Dosimetric verification of helical tomotherapy for total scalp irradiation. Medical physics 35, 5061-5068.

International Atomic Energy Agency, 2011. Status of Computed Tomography Dosimetry for Wide Cone Beam Scanners, IAEA Human Health Reports 5. 
Kwan, I.S., Wilkinson, D., Cutajar, D., Lerch, M., Rosenfeld, A., Howie, A., Bucci, J., Chin, Y., Perevertaylo, V.L., 2009. The effect of rectal heterogeneity on wall dose in high dose rate brachytherapy. Med Phys 36, 224-232.

Lian, C., Young, A., Cutajar, D., Freeman, N., Rosenfeld, A., 2012. Organ Point Dose Measurements in Clinical Multi Slice Computed Tomography (MSCT) examinations with the MOSkin Radiation Dosimeter. Rad Meas Special Issue, IWIRM 7 (Accepted).

Lian, C.P.L., Othman, M.A.R., Cutajar, D., Butson, M., Guatelli, S., Rosenfeld, A.B., 2011. Monte Carlo study of the energy response and depth dose water equivalence of the MOSkin radiation dosimeter at clinical kilovoltage photon energies. Australasian Physical \& Engineering Sciences in Medicine 34, 273-279.

Lin, P., Herrnsdorf, L., 2010. Pseudohelical scan for the dose profile measurements of 160mm-wide cone-beam MDCT. American Journal of Roentgenology 194, 897-902.

Mori, S., Endo, M., Nishizawa, K., Tsunoo, T., Aoyama, T., Fujiwara, H., Murase, K., 2005. Enlarged longitudinal dose profiles in cone-beam CT and the need for modified dosimetry. Med Phys 32, 1061-1069.

Nakonechny, K., Fallone, B., Rathee, S., 2005. Novel methods of measuring single scan dose profiles and cumulative dose in CT. Medical Physics 32, 98-109.

Rozenfeld, A.B., 2008 Radiation Sensor and Dosimeter (MOSkin) PCT/AU2008/000788 Australia.

Van der Molen, A., Geleijns, J., 2007. Overranging in multisection CT: quantification and relative contribution to dose- comparison of four 16-section CT scanners. Radiology 242, 208-216.

Wong, J.H.D., Carolan, M., Lerch, M.L.F., Petasecca, M., Khanna, S., Perevertaylo, V.L., Metcalfe, P., Rosenfeld, A.B., 2010. A silicon strip detector dose magnifying glass for IMRT dosimetry. Med Phys 37, 427-439.

Wong, J.H.D., Knittel, T., Downes, S., Carolan, M., Lerch, M.L.F., Petasecca, M., Perevertaylo, V.L., Metcalfe, P., Jackson, M., Rosenfeld, A.B., 2011. The use of a silicon strip detector dose magnifying glass in stereotactic radiotherapy QA and dosimetry. Med Phys 38, 1226-1238. 